\title{
CHARACTERISTICS OF STRIPLINES WITH INHOMOGENEOUS CYLINDRICAL SUBSTRATE
}

\author{
CHUNG-RUNG LEE, JEAN-FU KIANG, AND CHUN HSIUNG CHEN
}

\author{
Department of Electrical Engineering and Graduate Institute of Communication Engineering \\ National Taiwan University \\ No.1, Sec.4, Roosevelt Rd. \\ Taipei, Taiwan 10617, ROC \\ E-mail: jfkiang@cc.ee.ntu.edu.tw
}

\begin{abstract}
A mode-matching technique combined with Galerkin's method is proposed in this paper to analyze the characteristics of striplines embedded in an inhomogeneous cylindrical medium. The potential in each layer is expressed in terms of eigenmodes obtained numerically. Coupling between two sets of eigenmodes in contiguous layers are described by defining potential ratio coefficients. An integral equation is derived based on these eigenmodes with charge on the stripline as unknown. Method of moments is applied to solve this integral equation. Various parameters are analyzed for their effects on the transmission characteristics.
\end{abstract}

\section{Introduction}

Cylindrical striplines can be used in the feed networks of cylindrical antennas [1]. Various techniques have been used to analyze cylindrical transmission line problem. In [2], a mode-matching technique combined with Galerkin's method is used to calculate the capacitance matrix of striplines embedded in an inhomogeneous planar stratified medium modeling practical substrate materials. In [3], a modified residue calculus technique is applied to calculate the characteristic impedance of elliptic stripline in a layered medium. Similar structure was studied by using an iterative technique [4]. In [5], a variational technique in the spectral domain is developed to study arbitrary multiconductor and multidielectric cylindrical stripline configurations. In [6], a cylindrical microstrip line in an half-open space is studied. In [7], a conformal mapping technique is applied to calculate the capacitance matrix of elliptical microstrip line.

In all the above works, each layer is considered as a homogeneous medium. In this paper, we emphasize that the medium in each layer can be inhomogeneous in the azimuthal direction. Local inhomogeneities near the stripline can be utilized to adjust the phase velocities of the guided wave modes.

\section{Formulation}

Figure 1 shows the cross section of a uniform stripline embedded in an inhomogeneous cylindrical layered medium. The electrostatic field is derived from a potential distribution which satisfies the generalized Laplace equation. Using the separation-of-variable technique, the potential distribution is expressed as a summation of mode functions. These mode functions are in turn expressed as a superposition of the mode functions in a homogeneous layered medium. Potential ratio coefficients are defined to facilitate the derivation. Imposing the boundary conditions that the potential and the normal electric flux density are continuous across layer interfaces, recursive formulas for the potential ratio coefficients are obtained.

At the layer interface where the stripline is located, the potential distributions are continuous, and the discontinuity of normal electric flux density accounts for the charge distribution on the stripline. Thus, the potential can be expressed in terms of the charge distribution on the stripline. Finally, impose the condition that the potential on the stripline surface is equal to the implied voltage to form an integral 
equation with the charge distribution as unknown.

To solve the integral equation, first choose a set of basis functions to expand the surface charge density, then choose the same set of basis functions as weighting functions. Take the inner product of these weighting functions with the integral equation to obtain a matrix equation. The per-unit-length capacitance matrix and characteristic impedance of the transmission lines are then derived from the charge density distribution.

\section{Results and Discussions}

We first verify our approach by comparing the calculation results with those in the literatures. Figure 2 shows the characteristic impedance of a stripline inserted in a coaxial cable as shown in the inlet. It is shown that the numerical results converge as the mode number is greater than 60 . It is also found that more modes are needed to obtain convergent results when the basis number is increased. Using more modes implies better resolution of the charge distribution. Our convergent results match reasonably well with those in [3] and [4].

Next, investigate the effects of inhomogeneous media on the characteristics of striplines. Figure 3 shows the effective dielectric constant of a stripline in an inhomogeneous layer which encloses a homogeneous layer with relative dielectric constant $\varepsilon_{r}$. A region with a relatively higher dielectric constant tends to induce stronger electric field. Hence, the effective dielectric constant increases as $\varepsilon_{r}$ increases. Due to the wide free-space above the strip, the effective dielectric constant increases less slowly than $\varepsilon_{r}$. Hence, the range of the effective dielectric constant is smaller than the range of $\varepsilon_{r}$. It is observed that the effective dielectric constant is almost independent of the strip width and appears like a linear function of the substrate dielectric constant, similar to those in [6].

Figures 4 and 5 show the effective dielectric constant of the even mode for two coupled striplines supported by a thin membrane. The air pocket below the thin membrane is used to increase the phase velocity of the guided signal. In Figure 4, the air pocket extends between the inner edges of the two coupled striplines. It is observed that the effects become obvious when the pocket depth is larger than $0.01 \mathrm{R}$. The effective dielectric constant decreases as the two striplines are moved apart. More electric field lines pass through the air pocket when the air pocket depth increases. Thus, the effective dielectric constant is reduced.

In Figure 5, the air pocket extends between the outer edges of the striplines. It is observed that the effects become obvious when the pocket depth is larger than $0.001 \mathrm{R}$ rather than $0.01 \mathrm{R}$ as in Figure 4 . The effective dielectric constant is reduced more obviously than in the previous case because the air pocket is wider in this case. Also notice that the effective dielectric constant of two striplines with deep air pocket is smaller than the corresponding one in Figure 5. The effective dielectric constant of the odd modes for two coupled striplines is similar to those of the even modes. Similar results are also observed for the homogeneous striplines [7].

Figure 6 shows the deviation of the even and the odd modes for two coupled striplines with a block of high dielectric constant inserted in the substrate between the two lines. The deviation vanishes when the depth of the high permittivity region is varied from $0.001 \mathrm{R}$ to $0.01 \mathrm{R}$. This implies that the mode dispersion of this structure can be adjusted to zero. The deviation turns negative when the depth of the high permittivity region is increased from $0.1 \mathrm{R}$. As the channel thickness increases, more electric fields are confined between the two striplines through the channel. Thus, the effective dielectric constant of the odd mode increases more obviously than that of the even mode. As the bottom of the channel moves closer to the center conductor, more field lines tend to be attracted to the center conductor. Thus, the dielectric constant of the even mode increases more obviously with increasing depth of the high permittivity region than that of the odd mode. 


\section{Conclusions}

A mode-matching technique combined with Galerkin's method is proposed in this paper to study the characteristics of striplines embedded in a cylindrical layered medium inhomogeneous in the azimuthal direction. Several inhomogenous dielectric profiles are studied to understand the effects of inhomogeneities on the characteristic impedance and other related parameters like effective dielectric constant and phase velocity of the striplines.

\section{References}

[1] J.-H. Lu and K.-L. Wong, "Analysis of slot-coupled double-sided cylindrical microstrip lines," IEEE Trans. Microwave Theory Tech., vol.44, pp.1167-1170, July 1996.

[2] J.-F. Kiang, "Capacitance of microstrip lines with inhomogeneous substrate," IEEE Trans. Microwave Theory Tech., vol.44, pp.1703-1709, October 1996.

[3] K. K. Joshi and B. N. Das, "Analysis of elliptic and cylindrical striplines using Laplace's equation," IEEE Trans. Microwave Theory Tech., vol.28, pp.381-386, April 1980.

[4] C. H. Chan and R. Mittra, "Analysis of a class of cylindrical multiconductor transmission lines using an iterative approach," IEEE Trans. Microwave Theory Tech., vol.35, pp.415-424, April 1987.

[5] F. Medina and M. Horno, "Spectral and variational analysis of generalized cylindrical and elliptical strip and microstrip lines," IEEE Trans. Microwave Theory Tech., vol.38, pp.1287-1293, September 1990.

[6] H. A. Auda, "Cylindrical microstrip line partially embedded in a perfectly conducting ground plane," IEEE Trans. Microwave Theory Tech., vol.39, pp.1662-1666, September 1991.

[7] D. Homentcovschi, "A cylindrical multiconductor stripline-like microstrip transmission line," IEEE Trans. Microwave Theory Tech., vol.37, pp.497-503, March 1989. 


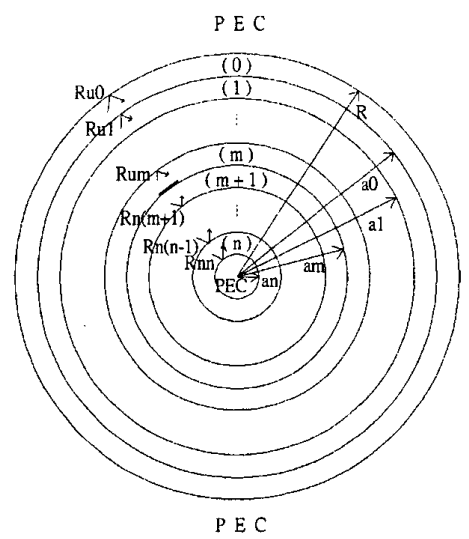

Fig.1 : Cross sectional view of a stripline embedded in a cylindrical inhomogeneous medium.

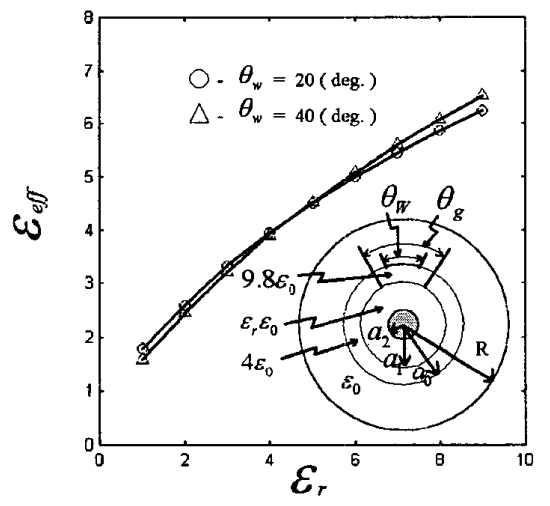

Fig.3 Effect of background $\mathcal{E}_{r}$ on the effective dielectric constant of a stripline on a segmented inhomogeneous substrate, $\theta_{8}=80^{\circ}$,

$a_{0} / R=0.6, a_{1} / R=17 / 30, a_{2} / R=0.5$.

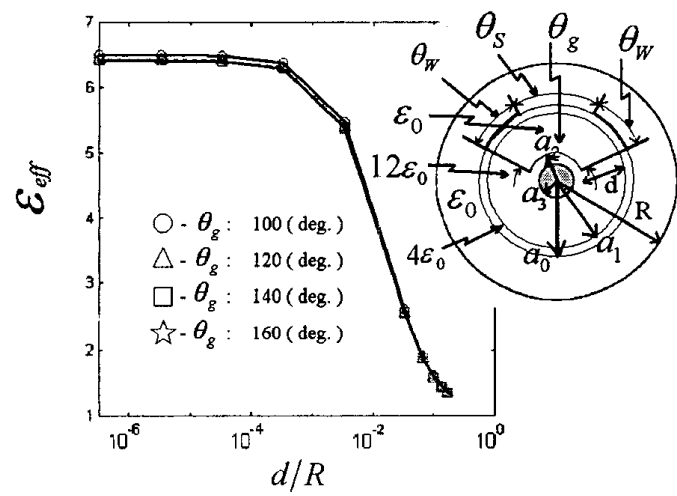

Fig.5 Effect of $d / R$ on the effective dielectric constant of the even mode for two coupled striplines, $\quad a_{0} / R=20.2 / 30, a_{1} / R=2 / 3$,

$$
a_{2} / R=0.5, \theta_{w}=40^{\circ} \text {. }
$$

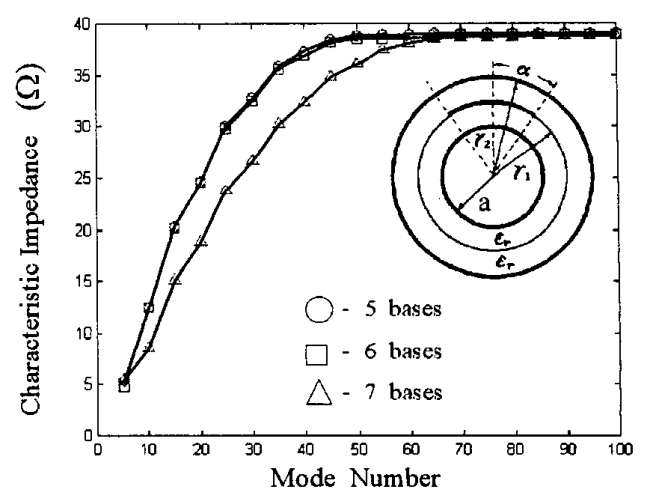

Fig.2 Variation of characteristic impedance with mode number, $\quad r_{1} / a=1.8, \quad r_{2} / a=2.0$, $\mathcal{E}_{r}=1.0, \quad \alpha=19.95^{\circ}$.

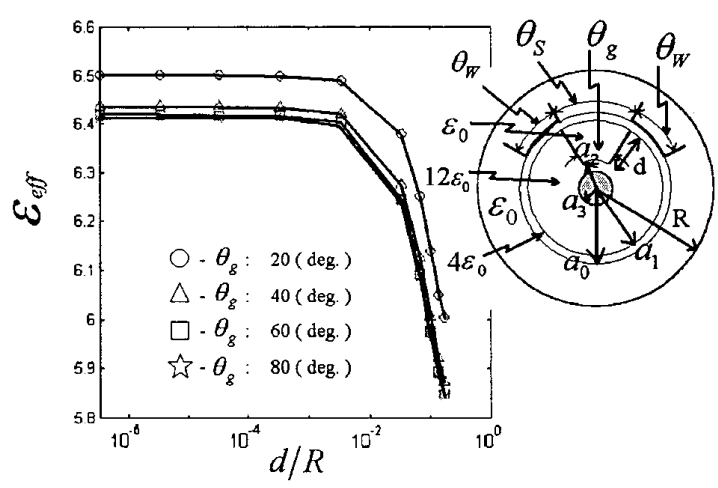

Fig.4 Effect of $d / R$ on the effective dielectric constant of the even mode for two coupled striplines, $\quad a_{0} / R=20.2 / 30, \quad a_{1} / R=2 / 3$, $a_{2} / R=0.5, \theta_{w}=40^{\circ}$.

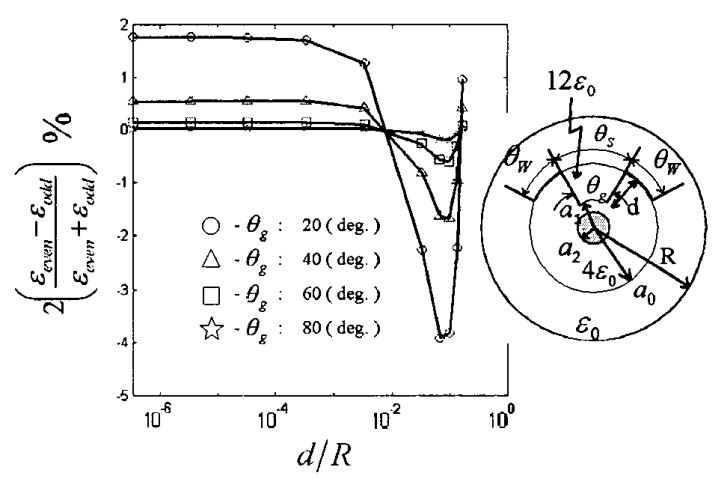

Fig.6 Effect of $d / R$ on the effective dielectric constants of the even and the odd modes for two coupled striplines, $a_{0} / R=2 / 3, a_{2} / R=0.5$,

$\theta_{w}=40^{\circ}$. 